Vol. 39(1), pp. 13-20, June 2020

ISSN 1821-536X (print)

ISSN 2619-8789 (electronic)
Tanzania Journal of Engineering and Technology Copyright (C) 2020 College of Engineering and

Technology, University of Dar es Salaam

Full Length Research Paper

\title{
Multipath Exploitation-Based Indoor Target Localization Model Using Single Marginal Antenna
}

\author{
Joseph Sisala ${ }^{1}$ and Abdi T. Abdalla ${ }^{2}$ \\ ${ }^{1}$ Electrical Engineering Department, University of Dar es Salaam, Tanzania \\ ${ }^{2}$ Electronics and Telecommunications Engineering Department, University of Dar es \\ Salaam, Tanzania \\ Corresponding Author: mwaki.joseph@udsm.ac.tz
}

\begin{abstract}
Recently, indoor target localization became an area of interest due to its diverse applications. In indoor target localization, surrounding environment creates multipath components, which can be exploited to aid in localization process. A number of studies have been proposed to employ multipath exploitation in localizing indoor targets. However, their localization errors can still be improved. This study proposed a new localization model based on multipath exploitation techniques by using triangulation method. Ultra-wide band signals were resolved and associated using marginal antenna-based scheme. The estimate of the target location was then obtained using measured round-trip time delays. The location was determined by applying the simple trigonometry on the triangle in which real radar, virtual radars, and the target location are the vertices of the triangle in question. Simulation results show that the proposed method has improved the localization error over a wide range of timing errors, target locations and room sizes with the overall maximum localization error of $1.4 \mathrm{~m}$ equivalent to $22.2 \%$ improvement as compared to $1.8 \mathrm{~m}$ localization error obtained using the method developed by the Muqaibel et al. (2017).
\end{abstract}

Keywords: Indoor target localization, marginal-antenna, multipath exploitation, virtual radar.

\section{INTRODUCTION}

Multipath exploitation in indoor target localization and imaging has drawn much attention in the past few years due to its ability to localize, track, and imaging an indoor target even in non-line of sight scenarios using only a single antenna (Setlur et al., 2010; Deiana et al., 2010; Setlur et al., 2011; Setlur et al., 2012; Smith and Mobasseri., 2012; Leigsnering et al., 2015; Muqaibel et al., 2014; Muqaibel et al., 2017). However, according to Setlur et al. (2013) and Amin and Ahmad (2013), multipath exploitation in indoor localization has not been extensively studied yet. The additional energy contained in multipath components may offer additional information on the obscured target (Lui and So, 2010; Chakraborty et al., 2010; Smith and Mobasseri, 2011; Smith and Mobasseri, 2012). 
Contrary to multi antenna or Synthetic Aperture Radar (SAR) localization, single antenna localization offers a number of advantages including reduced execution time and system portability (Setlur et al., 2010; Deiana et al., 2010; Setlur et al., 2011; Setlur et al., 2012; Smith and Mobasseri., 2012; Muqaibel et al., 2014; Leigsnering et al., 2015, Muqaibel et al., 2017). In Setlur et al., (2010), authors proposed a system that utilized multipath exploitation to improve tracking of moving objects in indoor scenarios. In Deiana et al., (2010), the authors developed a MultipleInput Multiple-Output (MIMO) radar system to detect the presence of a target in indoor environment.

In Setlur et al. (2012), authors developed an algorithm based on a single transceiver to localize the indoor target exploiting extra information from multipath returns. As an improvement of this work, same authors proposed a single-antenna localization method which uses antenna directivity to aid in the localization process, which reduced the number of possible wall multipath association from six down to four. In Muqaibel et al. (2017) the authors proposed a localization method using single marginal antenna, in which the complexity of the wall association algorithm was reduced greatly. This was possible since the contribution of one side wall was eliminated by strategically locating the transceiver taking into account the aspect dependence property of the multipath returns. However, the overall accuracy can still be improved to suit critical applications including the military.

The aspect dependence property holds that specular multipath components exist only when the radar is placed at a certain radar location. The target location is defined as the point of intersection of three radar loci: a circle due to direct return and other two circles due to virtual radars as mirrored from one side-wall and the back wall as a result of monostatic configurations. This paper proposes a novel indoor target localization method based on the multipath exploitation technique. The problem is reformulated using only first-order expressions which anticipated to reduce localization error significantly. The method adopts the wall-multipath association algorithm suggested by Muqaibel et al (2017).

Monostatic configuration was considered about the true and virtual radars. In this research, the target location was the intersection of straight lines. The underlined assumption is that the knowledge of reflecting geometry is either known in priori or can be easily estimated. The contribution of this paper is twofold, developed a novel and accurate indoor target localization model based on multipath exploitation and the paper provides mathematical expressions for localization error based on the developed model.

\section{MATERIALS AND METHODS}

The triangulation method of localization due to its nature of being less prone to higher order expressions as compared to conic sections and circles has been used in formulating the proposed method while MATLAB software due to its wider applicability on science and engineering research has been used to simulate the proposed model. This section is having two sub sections, the first subsection introduces the concept of multipath while the second subsection explains the developed method.

\section{Multipath signal model}

Consider a given scene in Figure 1 with a single physical radar, $R_{0}$, and three virtual radars (VR), $R_{i}, i=1,2,3$ as results of respective walls' reflections. VR is created 
when the signal is reflected from the interior wall, where the return path assumes an alternative antenna-target configuration (Muqaibel et al., 2017). Wall-1 is located along crossrange, $x=0$ and the transceiver is placed along downrange, $y=0$ at a standoff distance, $\mathrm{d}_{\mathrm{f}}$. The size of the room was taken to be $D_{1} x D_{2} \mathrm{~m}^{2}$. Let $\tau_{0}$ represents forward trip time delay from $R_{0}$ to the unknown target located at $(x, y)$.

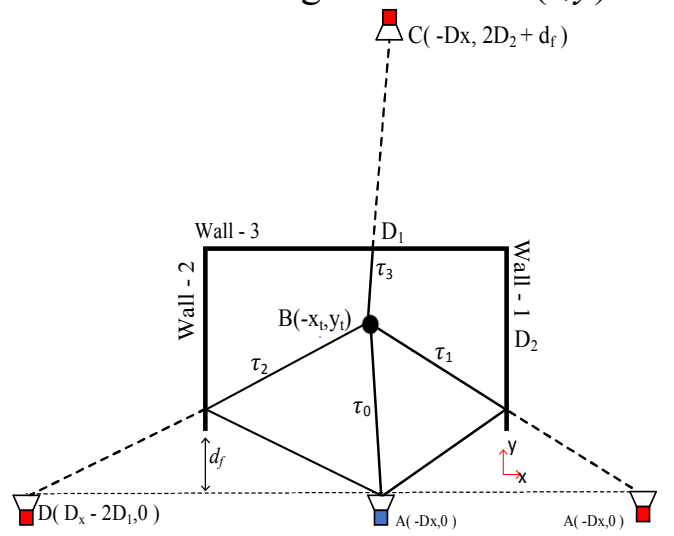

Figure 1: Multipath scene model with virtual radars (Muqaibel et al., 2014)

Assuming the surrounding walls are smooth and the transmitted signal, $s(t)$, is resolvable in which $R$ returns are registered by the radar system. If the radar system transmits a UWB signal, then the received signal, $y(t)$, under Additive Gaussian Noise (AGN), $v(t)$, is given by (Muqaibel et al., 2017).

$$
y(t)=\sum_{i=0}^{R-1} A_{i} s\left(t-t_{i}\right)+A_{w} s\left(t-t_{w}\right)+v(t)
$$

Where $A_{i}$ and $t_{i}$ represent the $i^{\text {th }}$ amplitude and round-trip delay of the $i^{\text {th }}$ multipath return, respectively. In the second term, $A_{w}$ and $t_{w}$ represent amplitude and time delay of the front wall contribution. The time delay from the target to $R_{i}$ associated with wall- $i$ is therefore, given by $\tau_{i}=t_{i}-t_{0} / 2, i>0$. As depicted in Figure 1, there are at most four signal returns; direct return and three first-order multipath from the interior walls. This work adopts the radar configuration as well as wall association suggested by Muqaibel et al. (2017).

\section{Proposed Localization Method}

Multipath are aspect dependent and they may be observed only at certain radar locations. This characteristic can be utilized to aid in optimally locating the radar system such that the multipath effect is minimized. The optimal radar location is adopted from the marginal radar configuration as suggested in Muqaibel et al. (2017). The configuration in (Muqaibel et al., 2017) depicted in Figure 2 uses the fact that multipath returns are aspect dependent making the marginal antenna configuration receive a limited number of returns and hence reduces the complexity of associating multipaths to the corresponding wall. UltraWide Band (UWB) signal of bandwidth 2 $\mathrm{GHz}$ was transmitted and received by the radar located along the right wall at a standoff distance of $2 \mathrm{~m}$.

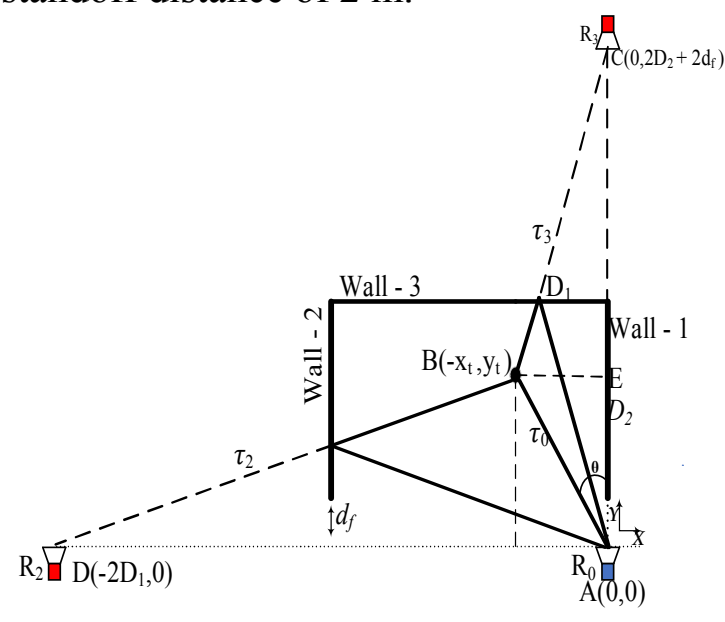

Figure 2: Single Marginal radar configuration scene model

Since the direct and back return always exist, the proposed method will make use of $\tau_{0}$ and $\tau_{3}$. The first step is to estimate $\angle B A C$ by considering $\triangle A B C$. The length of the side $\overrightarrow{A B}$ is $c \tau_{0}, \overrightarrow{B C}$ is $c \tau_{3}$ and 
$\overrightarrow{A C}$ is $2 D_{2}+2 d_{f}$. Applying cosine rule, we have:

$$
\angle B A C=\cos ^{-1}\left[\frac{(A B)^{2}+(A C)^{2}-(B C)^{2}}{2(A B)(A C)}\right] \ldots . .
$$

where $c$ is the speed of electromagnetic wave in free space, $D_{1}$ and $D_{2}$ are the lengths of room along with the crossrange and downrange directions, respectively. The target is located at point $B$ at the vertices of the triangle $\triangle A B C$ depicted in Figure 3, the other vertices $A$ and $C$ are at $R_{0}$ (Position of the real radar) and $R_{3}$ (Position of the virtual radar as mirrored from the back wall), respectively. Target location is obtained by resolving the direct path vertically and horizontally as shown in equations (3) and (4).

$x_{t}=-c \tau_{o} \sin (B A C)$

$$
y_{t}=c \tau_{o} \cos (B A C)
$$

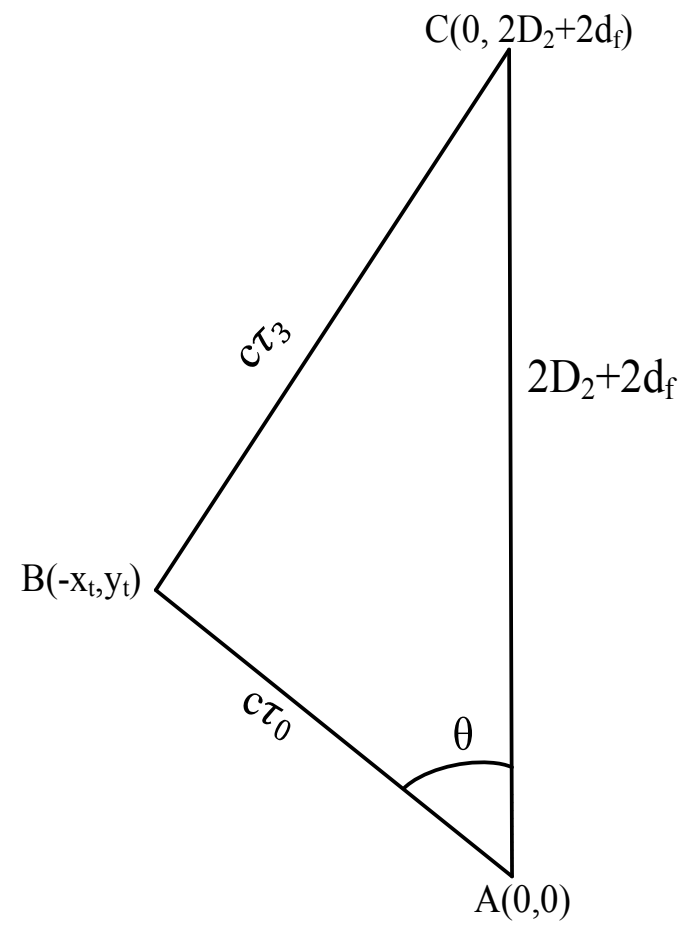

Figure 3: Triangle used to formulate the proposed Method

\section{RESULTS AND DISCUSSION}

Simulating the scene model in Figure 2, with a target randomly located at $(-20,3)$ $\mathrm{m}$. The right and left sidewalls are located at the origin and at a crossrange of $-25 \mathrm{~m}$, respectively. The back wall resides at $10 \mathrm{~m}$ in the downrange. The received signal was pre-processed to mitigate the contribution of the front wall as in (Yoon and Amin., 2009; Tivive et al., 2011; Lagunas et al., 2012; Tivive et al., 2014). The direct return, the first and second reflections associated with the two walls were recorded. The time delay between the radar and the target, $\tau_{0}=67.41 n s$, the time delay associated with the two VRs, $\tau_{2}=105.2 n s$ and $\tau_{3}=96.676 n s$. By invoking the wallmultipath association algorithm developed by Muqaibel et al. (2017), multipath delays were perfectly associated to their respective walls and by using the proposed model the target was localized as shown in Figure 4.

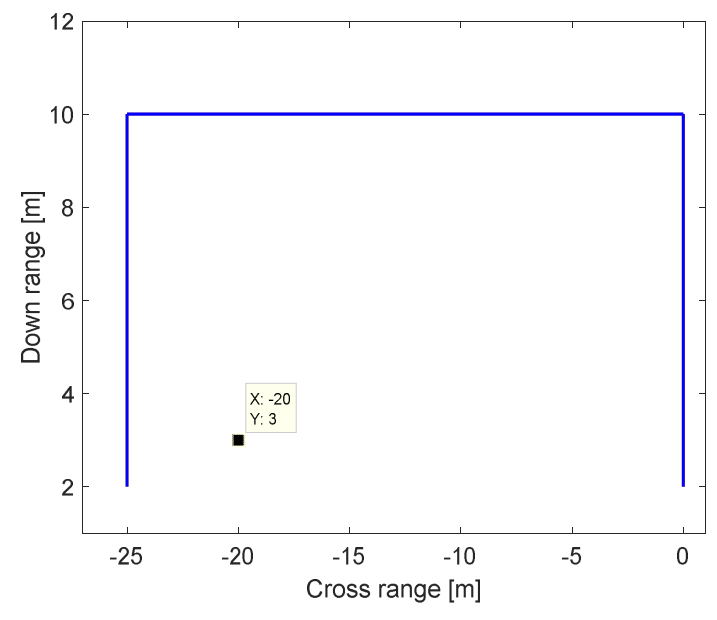

Figure 4: Target Localization with the Proposed Model

\section{Sensitivity to timing errors}

To evaluate the performance of the proposed scheme, 50 randomly generated targets were localized. During simulation, a range of timing error due to limited bandwidth and SNR were assumed be from $-2 n s$ to $2 n s$ to investigate the accuracy and robustness of the proposed model. In 
this study, localization error was used to quantify the performance of the proposed method.

Let $\Delta \tau_{0}$, and $\Delta \tau_{3}$ represent the timing errors associated with direct return, and return from the back wall, respectively. Suppose that the estimated target location is $\left(x_{t}+\Delta x_{t}, y_{t}+\Delta y_{t}\right)$, then the possible error along the crossrange direction is given by equation (5) in accordance with Muqaibel et al. (2017).

$$
\Delta x_{t}=\left|\frac{\partial x_{t}}{\partial \tau_{0}}\right| \Delta \tau_{0}+\left|\frac{\partial x_{t}}{\partial \tau_{3}}\right| \Delta \tau_{3}
$$

Similarly, the possible downrange error is given by equation (6).

$$
\Delta x_{t}=\left|\frac{\partial x_{t}}{\partial \tau_{0}}\right| \Delta \tau_{0}+\left|\frac{\partial x_{t}}{\partial \tau_{3}}\right| \Delta \tau_{3}
$$

From equations (5) and (6), the maximum localization error, $\Delta r$, which is the displacement of the estimated target location from the exact location, is given by equation (7).

$$
\Delta r=\sqrt{\Delta x_{t}^{2}+\Delta y_{t}^{2}}
$$

The expressions for the partial derivatives in equations (5) and (6) were derived as:

$$
\begin{aligned}
& \frac{\partial x_{t}}{\partial \tau_{0}}=\frac{\partial x_{t}}{\partial z} \times \frac{\partial z}{\partial \tau_{0}} \\
& \frac{\partial x_{t}}{\partial \tau_{3}}=\frac{\partial x_{t}}{\partial z} \times \frac{\partial z}{\partial \tau_{3}} \\
& \frac{\partial x_{t}}{\partial z}=\frac{1}{8 \sqrt{z}\left(D_{2}+D_{f}\right)} \\
& \frac{\partial z}{\partial \tau_{0}}=32 c^{2} \tau_{0}\left(D_{2}+d_{f}\right)^{2}-2 m \frac{\partial m}{\partial \tau_{0}} \\
& \frac{\partial z}{\partial \tau_{3}}=4 c^{2} \tau_{3} m
\end{aligned}
$$

$$
\begin{aligned}
& \frac{\partial m}{\partial \tau_{0}}=2 c^{2} \tau_{0} \\
& \frac{\partial m}{\partial \tau_{3}}=-2 c^{2} \tau_{3} \\
& m=\left(c \tau_{0}\right)^{2}+\left(2\left(D_{2}+d_{f}\right)\right)^{2}-\left(c \tau_{3}\right)^{2} \\
& z=16\left(c \tau_{0}\right)^{2}\left(2\left(D_{2}+d_{f}\right)\right)^{2}-\left(\left(c \tau_{0}\right)^{2}+\left(2\left(D_{2}+d_{f}\right)\right)^{2}-\left(c \tau_{0}\right)^{2}\right)^{2} \\
& \frac{\partial y_{t}}{\partial \tau_{0}}=\frac{c^{2}}{2\left(D_{2}+D_{f}\right)} \tau_{0} \\
& \frac{\partial y_{t}}{\partial \tau_{3}}=-\frac{c^{2}}{2\left(D_{2}+D_{f}\right)} \tau_{3}
\end{aligned}
$$

From the equations (8) through (18), we can also deduce that we can localize more accurately when the room is large that is why the room size was chosen to be $25 \times 10$ m.

Figures 5(a) and 5(b) show the variations of the localization error with the timing errors associated with the side and back walls for the existing and proposed localization model, respectively. It can be observed that over a wide range of timing errors, the proposed localization model shows significant improvement from $1.8 \mathrm{~m}$ maximum localization error to $1.4 \mathrm{~m}$, which is equivalent to $22.2 \%$ compared to the existing model as suggested by Muqaibel et al. (2017). The localization error is more pronounced when the timing errors change in the same directions. 


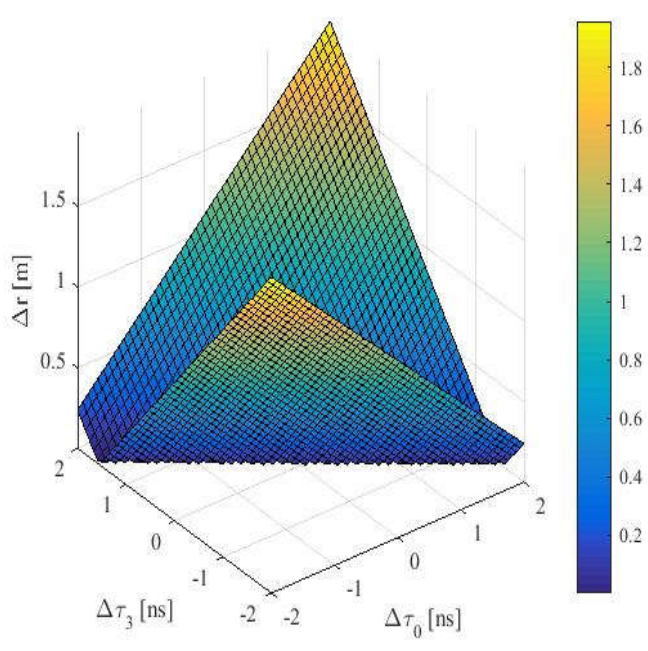

(a)

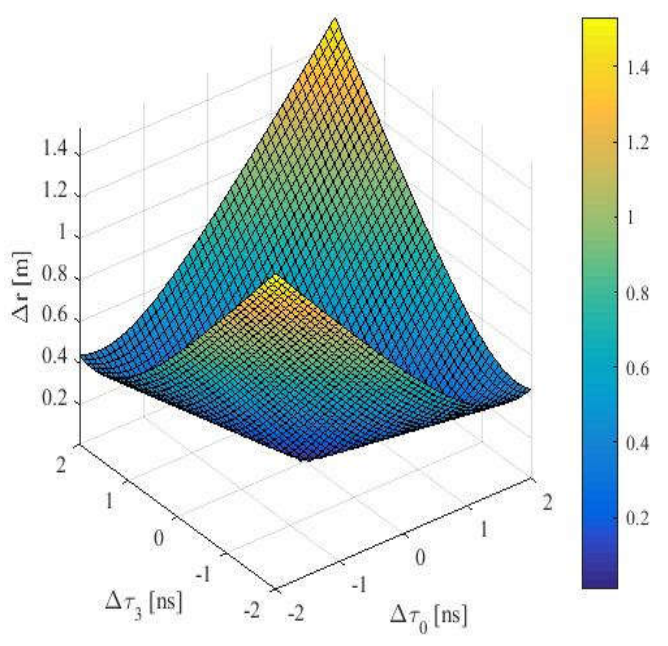

(b)

Figure 5: Variation of Localization errors with timing errors (a) Existing (b) Proposed

\section{Localization error with room size}

\section{A. Downrange direction}

In observing the variation of room dimensions along the downrange variation, the crossrange dimension was held fixed at $25 \mathrm{~m}$ while varying the downrange from 4 to $10 \mathrm{~m}$ with the target location being fixed at $(-20,3) \mathrm{m}$. Simulation results are depicted in Figure 6, which shows that the performance of the two models vary together as their error functions nearly the same. When the downrange value is between 5 and $6 \mathrm{~m}$ the two models show the same performance. However, the performance of the proposed model is relatively better when the down range value reaches $6 m$ and above.

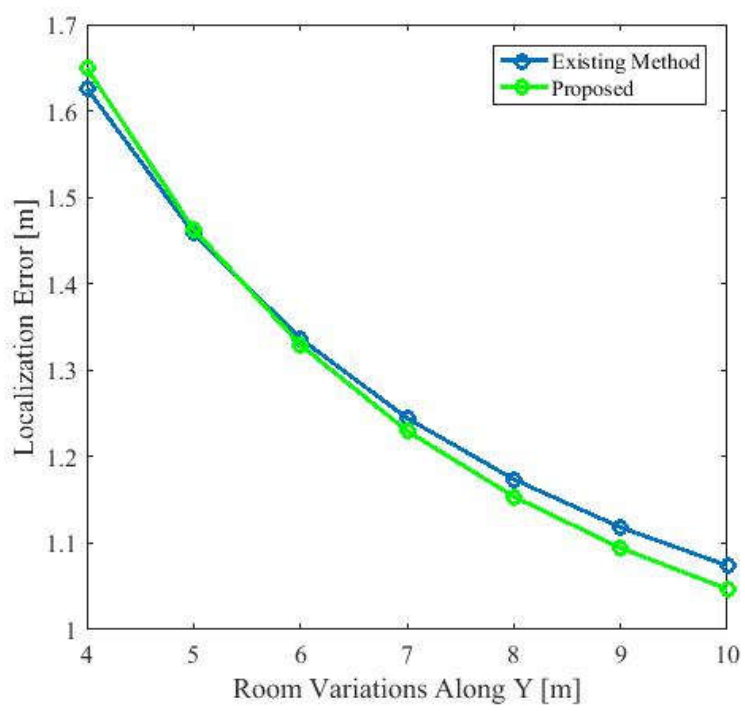

Figure 6: Localization error with room dimension in downrange

\section{B. Crossrange direction}

In observing the effect of room dimension along the cross-range, the downrange dimension was held fixed at $10 \mathrm{~m}$ while varying the cross-range from 5 to $25 \mathrm{~m}$. The target location was fixed at $(-20,3) \mathrm{m}$. The performance of proposed model is depicted in Figure 7, which shows that the localization errors of the existing and that of the proposed model. From the results in Figure 7, the proposed model shows some improvement from $1.175 \mathrm{~m}$ timing errors to $1.155 \mathrm{~m}$, which is equivalent to $1.73 \%$. 


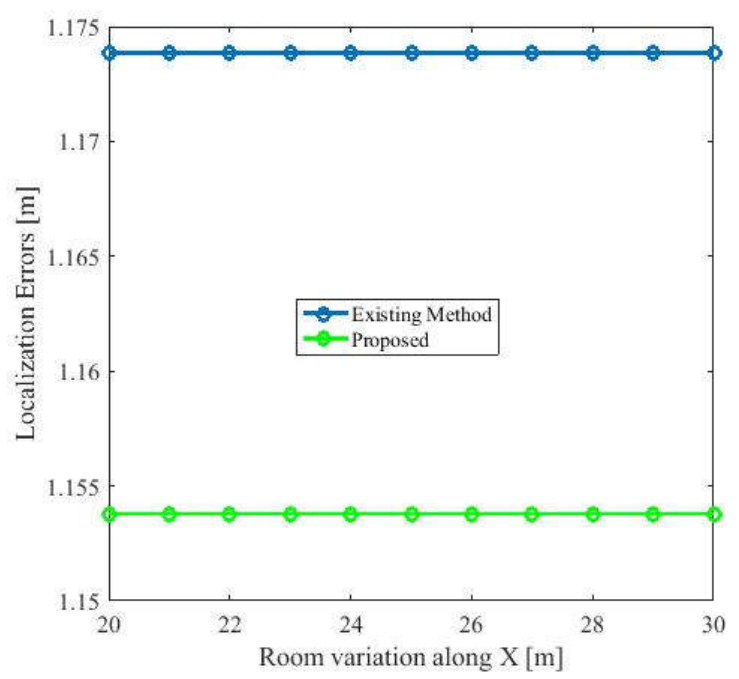

Figure 7: Localization error with room dimension in crossrange

\section{CONCLUSIONS}

In this paper, a new localization method based on multipath exploitation and single marginal antenna was proposed. Target localization was made possible by considering a monostatic configuration with triangulation approach. The expressions for the localization errors as functions of the timing errors were derived and analyzed under different localization scenarios. Simulation results showed that the proposed model has better performance, in terms of localization error, for a wide range of timing errors, room sizes and target locations. As the extension of this work, the study can be improved to tracking a moving target.

\section{ACKNOWLEDGEMENTS}

This work was funded by the University of Dar es Salaam Competetive Projects, through the directorate of research, award number COICT-ETE 19048.

\section{REFERENCES}

Amin M.G. and Ahmad F. (2013). Compressive Sensing for Through-The-
Wall Radar Imaging. Journal of Electronic Imaging, 22(3): 030901.

Chakraborty B., Li Y., Zhang J.J., Trueblood T., Papandreou-Suppappola A. and Morrell D. (2010). Multipath exploitation with adaptive wave form design for tracking in urban terrain. In March 2010 IEEE International Conference on Acoustics, Speech and Signal Processing, 3894-3897. DOI: $10.1109 /$ ICASSP.2010.5495808

Deiana D., Kossen A.S. and Van Rossum W.L. (2010). Multipath Exploitation in An Urban Environment Using A MIMO Surveillance Radar. In June $201011^{\text {th }}$ International Radar Symposium, 1-4. Article number 5547449.

Lagunas E., Amin M.G., Ahmad F. and Nájar M. (2012). Wall Mitigation Techniques for Indoor Sensing Within the Compressive Sensing Framework. In 2012 IEEE 7th Sensor Array and Multichannel Signal Processing Workshop (SAM), 213-216. DOI: $\underline{\text { 10.1109/SAM.2012.6250470 }}$

Leigsnering M., Ahmad F., Amin M.G. and Zoubir A.M. (2015). Multipath exploitation in sparse scene recovery using sensing-through-wall distributed radar sensor configurations. In Proceedings of ICASSP, IEEE International Conference on Acoustics, Speech and Signal Processing. https://doi.org/10.1109/ICASSP.2015.71 7847.

Lui K.W. and So H.C. (2010). Range-Based Source Localization With Pure Reflector in Presence of Multipath Propagation. Electronics Letters, 46(13): 957-958. DOI: $\underline{10.1049 / \mathrm{el} .2010 .3431}$

Muqaibel A.H., Amin M.G. and Ahmad F. (2014). Directional multipath exploitation for stationary target localization with a single-antenna. In Int. Radar Conf. Catch. Invis., Lille, France.

Muqaibel A.H., Abdalla A.T. and Alkhodary M.T. (2017). Indoor Target 
Localization Using Single Marginal Antenna With Virtual Radars Support. International Journal of Microwave and Wireless Technologies, 9(9): 1863-1870. DOI: https://doi.org/10.1017/S17590787 17000769

Setlur P., Amin M. and Ahmad F. (2010). Multipath Doppler Signatures from Targets Moving Behind Walls. In May 2010 IEEE Radar Conference, 799-803.

Setlur P., Amin M. and Ahmad F. (2011). Multipath Model and Exploitation in Through-The-Wall and Urban Radar Sensing. IEEE Transactions on Geoscience and Remote Sensing, 49(10): 4021-4034. DOI: 10.1109/TGRS.2011.2128331

Setlur P., Smith G.E., Ahmad F. and Amin M.G. (2012). Target Localization with a Single Sensor via Multipath Exploitation. IEEE Transactions on Aerospace and Electronic Systems, 48(3): $\quad$ 1996-2014. DOI: $10.1109 /$ TAES.2012.6237575

Setlur P., Alli G. and Nuzzo L. (2013). Multipath Exploitation in Through-Wall Radar Imaging via Point Spread Functions. IEEE Transactions on Image Processing, 22(12), 4571-4586. DOI: $\underline{10.1109 / T I P .2013 .2256916}$
Smith G.E. and Mobasseri B.G. (2011). Robust Through-The-Wall Radar Image Classification Using a Target-Model Alignment Procedure. IEEE Transactions on Image Processing, 21(2): $\quad$ 754-767. DOI: $10.1109 /$ TIP.2011.2166967

Smith G.E. and Mobasseri B.G. (2012). Multipath Exploitation for Radar Target Classification. In May 2012 IEEE Radar Conference, 0623-0628.

Tivive F.H.C., Amin M.G. and Bouzerdoum A. (2011). Wall Clutter Mitigation Based on Eigen-Analysis in Through-The-Wall Radar Imaging. In $201117^{\text {th }}$ International Conference on Digital Signal Processing (DSP), 1-8. DOI: $10.1109 /$ ICDSP.2011.6004992

Tivive F.H.C., Bouzerdoum A. and Amin M.G. (2014). A Subspace Projection Approach for Wall Clutter Mitigation in Through-The-Wall Radar Imaging. IEEE Transactions on Geoscience and Remote Sensing, 53(4): 2108-2122.

Yoon Y.S. and Amin M.G. (2009). Spatial Filtering for Wall-Clutter Mitigation in Through-The-Wall Radar Imaging. IEEE Transactions on Geoscience and Remote Sensing, 47(9): 3192-3208. DOI:10.1109/TGRS.2009.2019728 